\title{
Histologic transformation from lung squamous cell carcinoma in primary lesion to small cell lung cancer in metastatic lymph node lesion following lobectomy and adjuvant chemotherapy: a case report
}

\author{
Xinran Zhao, MD, MS ${ }^{a, b}$, Ye Qin, MD, MS ${ }^{a, b}$, Di Zhang, MDª, Yang Han, MDª Guangping Wu, MD,
} Huanyu Zhao, MD ${ }^{\mathrm{a}, \star}$

\begin{abstract}
Introduction: Lung cancer is the leading cause of cancer death worldwide. Histologic transformation from lung squamous cell carcinoma to small cell lung cancer (SCLC) has become more and more, and its treatment is challenging because of unknown pathogenesis.

Presentation of Case: A 62-year-old male patient presented with histologic transformation from primary lesion to metastatic recurrence lesion within 3 years following primary lobectomy and adjuvant chemotherapy. The patient had the symptom of cough at the time of initial illness and recurrence. The primary lesion at the root segment of left superior lobe was diagnosed as lung squamous cell carcinoma without gene mutation (including EGFR, ALK, BRAF V600E, etc.), and recurrence lesion at the swollen lymph nodes in mediastinum (4L group) was diagnosed as SCLC. Up to now, the patient was alive for nearly 4 years after first surgery.

Discussion: Surgical resection is the main treatment for lung cancer patient in early stage. Lymph node metastasis is a big threat for the patients in early stage. Recently, the research reports focused on the mechanism and cases of histologic transformation of lung cancer have become more and more. In this case, histologic transformation from squamous cell carcinoma in primary focus to SCLC in metastatic lymph node lesion following lobectomy and adjuvant chemotherapy. However, there was no gene mutation. This is a very unusual report.

Conclusion: We analyzed a lung cancer patient with histologic transformation from primary focus to lymphatic metastasis by clinical symptoms, radiologic features, laboratory tests, gene detection, histopathologic characteristics, and immunohistochemical testing. It is helpful to understand the pathogenesis of histologic transformation and metastatic progression for optimizing treatment measures to lung cancer.
\end{abstract}

Keywords: Histologic transformation, Squamous cell carcinoma, Small cell lung cancer, Lymphatic metastasis, Chemotherapy

\footnotetext{
a Department of Pathology, The First Affiliated Hospital and College of Basic Medical Sciences and ${ }^{b}$ Department of Medical Laboratory Technology, China Medical University, Shenyang, Liaoning, China

$X . Z$. and Y.Q. contributed equally to this work.

This manuscript has been peer reviewed.

Sponsorships or competing interests that may be relevant to content are disclosed at the end of this article.

*Corresponding author. Address: Department of Pathology, The First Affiliated Hospital and College of Basic Medical Sciences, China Medical University, No. 155 Nanjing North Street, Heping District, Shenyang 110001, Liaoning, China. Tel: 86-24-83282177. E-mail address: zhaohy@cmu.edu.cn (H. Zhao).

Copyright (C) 2020 The Authors. Published by Wolters Kluwer Health, Inc. on behalf of IJS Publishing Group Ltd. This is an open-access article distributed under the terms of the Creative Commons Attribution-Non Commercial-No Derivatives License 4.0 (CCBY-NC-ND), where it is permissible to download and share the work provided it is properly cited. The work cannot be changed in any way or used commercially without permission from the journal.

International Journal of Surgery Oncology (2020) 5:e93

Received 17 July 2020; Accepted 5 August 2020

Published online 26 October 2020
}

http://dx.doi.org/10.1097//J9.0000000000000093

\section{Key points}

- Histologic transformation from primary lesion to metastatic recurrence lesion.

- Histologic transformation from squamous cell carcinoma to small cell lung cancer (SCLC).

- Histologic transformation without gene mutation.

\section{Introduction}

Lung cancer is the leading cause of cancer death worldwide, and the recurrence and metastasis are the main threats to human beings. Even after complete resection of lung cancer patients with lymph node negative, recurrence will occur in the first 2 years ${ }^{[1,2]}$. The presence of lymph node metastasis has long been correlated with worsened clinical outcomes in lung cancer patients ${ }^{[3]}$.

According to World Health Organization (WHO) classification, the histologic type of lung cancer includes non-small cell lung cancer (NSCLC) $(80 \%-85 \%)$ and SCLC. and the main histologic type of NSCLC is adenocarcinoma and squamous cell 
carcinoma. In recent years, there are more and more cases of lung cancer with histologic transformation. Most of them are associated with EGFR mutation in the presence of histologic transformation from adenocarcinoma to SCLC ${ }^{[4]}$.

Here, we present a peculiar case with histologic transformation from lung squamous cell carcinoma in primary lesion to SCLC in metastatic lymph node lesion following lobectomy and adjuvant chemotherapy, and no gene mutation. We followed up by phone. Up to now, the patient was alive for nearly 4 years.

\section{Case report}

The patient is a Chinese man. He, 62 years old, is a factory worker, who was diagnosed with coronary disease and diabetes mellitus in 2012. He received insulin treatment for many years. This patient was firstly admitted to the First Affiliated Hospital of China Medical University in June, 2016. Before hospitalization, he had the symptom of cough and expectoration for more than 2 months. Occasionally, he had bloody sputum, but he did not have the symptom of chest distress, chest pain and dyspnea. And his diet, defecation and weight were normal. Computed tomography scan in grassroots hospital showed a space-occupying lesion in left superior lobe of lung.

Hospital examination showed that bilateral thoracic respiratory movement was consistent; the lung percussion was voiceless, no dry and wet rales. Percussion: no liver and kidney pain, no shifting dullness; auscultation examination: bowel sounds normal, no splashing sound; vertebral region: no tenderness and tapping pain. The following signs were all negative, including Babinski's sign, ankle clonus, Hoffmann sign, Kernig sign, Brudzinski sign, asterixis.

Abnormal laboratory results were as following (normal range in brackets). PH: 7.297 (7.350-7.450); $\mathrm{pCO}_{2}(\mathrm{a}): 52.4 \mathrm{~mm} \mathrm{Hg}$ (35.0-48.0); $\mathrm{pO}_{2}$ (a) $125.0 \mathrm{~mm} \mathrm{Hg}(83.0-108.0)$; $\mathrm{CHCO}_{3}$ (aP, st): $22.4 \mathrm{mmol} / \mathrm{L}(22.5-26.9)$; $\mathrm{ctCO}_{2}: 20.9 \mathrm{mmol} / \mathrm{L}$ (24.0-32.0); cBase (B): $-2.4 \mathrm{mmol} / \mathrm{L}(-2.0$ to 3.0$)$; CEA: $4.79 \mathrm{ng} / \mathrm{mL}(0.00-4.30)$; CA19-9: $32.15 \mathrm{U} / \mathrm{mL} \quad(0.00-27.00)$; Cyfra21-1: $3.49 \mathrm{ng} / \mathrm{mL}$ (0.00-3.30); plasma fibrinogen: $4.53 \mathrm{~g} / \mathrm{L}(2.00-4.00)$.

Direct detector chest radiography showed symmetrical chest and increased lung markings with a high density shadow $(3.9 \times 3.0 \mathrm{~cm}$ in size $)$ in the left middle lung near the heart border. There were micronodular shadows in apex of right lung and left costophrenic angle. The cardiac size and shape had no different change, and the costophrenic angle was sharp (Figs. 1A, B).

Emission computed tomography showed the concentration area of imaging agent distribution was in the anterior aspect of the left seventh and eighth ribs, the right sternoclavicular joint and the right forearm; there was no abnormal thickening, thinning, and defect area of imaging agent distribution in the rest of body bone. This indicated that bone metabolism was more active in these areas than that in other areas (Fig. 1C).

Pulmonary ventilation/perfusion imaging showed the abnormal sparseness and defect distribution of imaging agent in lingular segment of superior lobe and dorsal segment of left inferior lobe. Perfusion ratio: left lung/(left lung + right lung) $=37.33 \%$, right lung/(left lung + right lung $)=62.67 \%$. Above result showed that there was decreased pulmonary ventilation in these segments of left lung.

The patient was operated in the First Affiliated Hospital of China Medical University. The tumor was located at the root segment of left superior lobe, between left superior and left inferior lobar bronchi, which invaded the left superior lobar bronchial wall. The doctor cut off the root of left superior lobar bronchus and excised the left superior lobe, and lymph node $10-11$ groups were removed. The tumor $3 \times 3 \mathrm{~cm}$ in size was gray white. The result of pathologic examination was lung squamous cell carcinoma (well-moderate differentiated) with metastatic lymph node lesion around the splenic hilum (Station No. 10). For the microscopic characteristics of this surgical specimen, the cancer cells array as nest, and the nuclear is big, deep-stained. Pathologic karyokinesis can be seen (Fig. 2). TNM stage is T2N2M0.

During nearly 4 years of follow-up, the patient had received multiple cycles of chemotherapy. In August 2019, the patient had the symptom of cough and intermittent chest pain without obvious inducement for 20 days. Computed tomography scan in the emergency department of our hospital showed that irregular mass shadow was scattered in the left lung, and the lesion was close to the pleura (Figs. 3A, B); there was the enlargement of mediastinal lymph nodes (Figs. 3C, D). The cranial magnetic resonance imaging showed that there was no abnormal lesion in brain. Endobronchial ultrasound-guided transbronchial needle aspiration was performed for the swollen lymph nodes in mediastinum (4L group).

\section{Method}

We used $10 \%$ formalin to the pathologic tissues (the swollen lymph nodes in mediastinum) and embedded them in paraffin. Then we performed serial section $(4 \mu \mathrm{m})$ to each paraffin block and used hematoxylin-eosin and immunohistochemistry staining. Streptavidinperoxidase system (Ultrasensitive; Mai Xin, Fuzhou, China) was used for immunohistochemistry staining. The tissues were stained with the following prmary antibodies: broad-spectrum cytokeratin (pan-CK), CD56, TTF-1, Synaptophysin (Syn), Chromogranin A (CgA), p63, and Ki67. We replaced the primary antibody with phosphate buffered saline for the negative control. Written informed consent was obtained from the patient for publication of this case report and accompanying images. A copy of the written consent is available for review by the Editor-in-Chief of this journal on request. This work has been reported in line with the SCARE 2018 criteria $^{[5]}$.

\section{Result}

For the microscopic characteristics of the tissue punctured by endobronchial ultrasound-guided transbronchial needle aspiration, there were some lesions in the context of inflammatory exudation and bleeding, including a large number of atypical cells. The atypical cells were densely arranged, deeply stained, with great difference in shape and size. The nuclei were deeply stained, the cytoplasm was rare, and the ratio of nucleus to cytoplasm was very high (Figs. 4A, B).

Immunohistochemically, the tumor cells were diffusely positive for pan-CK, CD56, and Syn, weakly positive for CgA (cytoplasm staining). Positive nuclear expression of TTF-1 was detected in the tumor cells. Ki67 index was about 75\%. p63 was negative in tumor cells (Figs. 4C-I). Immunohistochemical results were consistent with SCLC. The diagnosis was made by 2 associate chief physicians. 

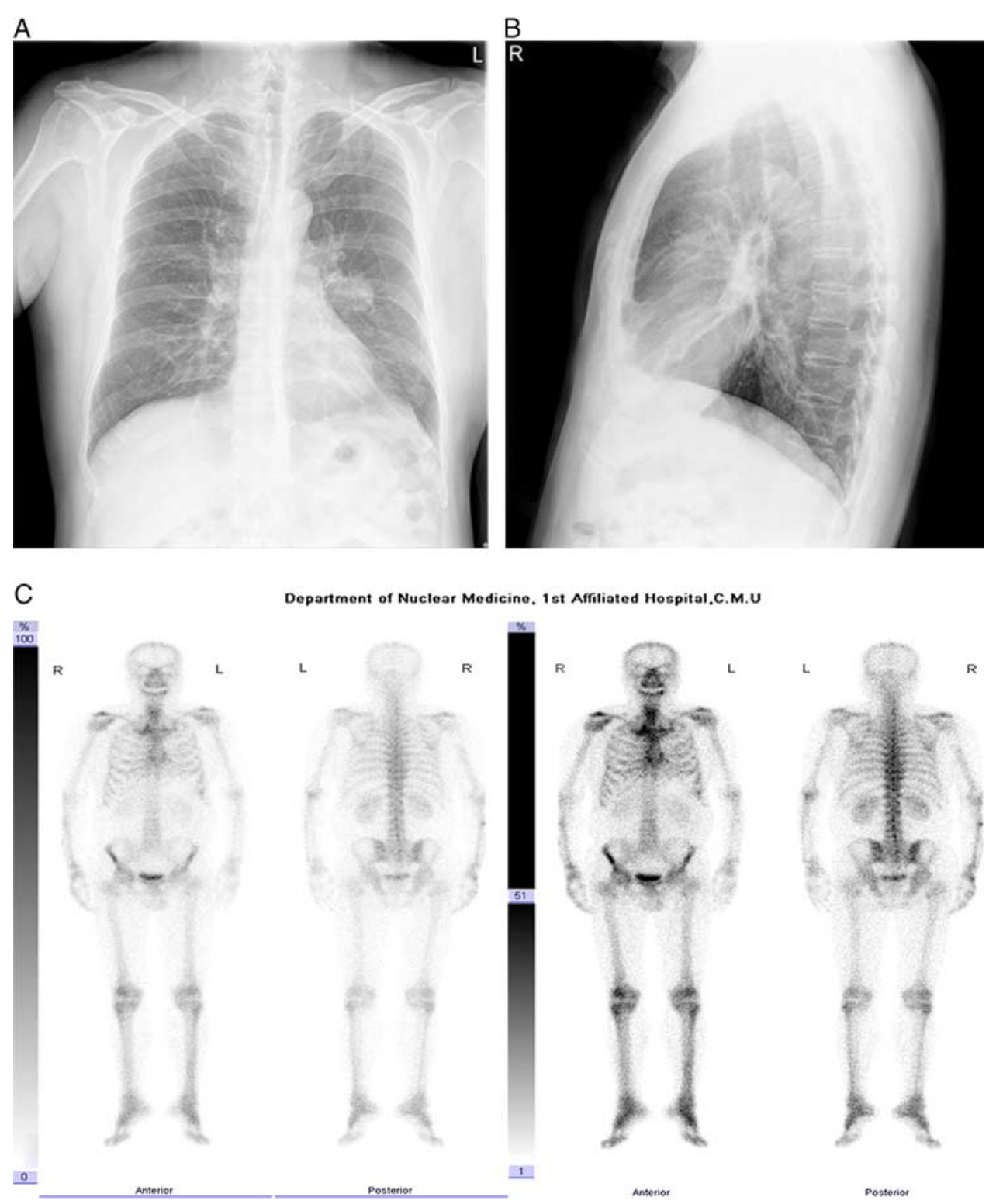

Figure 1. Imaging findings. A and B, Direct detector image. C, Emission computed tomography image.

A series of gene mutations were detected in surgical specimens of the first operation (lung squamous cell carcinoma) as following: MET exon 14-skipping mutation, KRAS exon 2 mutation, NRAS exon 3 mutation, PIK3CA gene mutation, BRAF V600E gene mutation, HER2 exon 20 mutation, the fusion of ALK, ROS1, and RET genes, EGFR mutation (exon 19 deletion mutation, exon 18 G719X point mutation, exon 20 S768I point mutation, exon $20 \mathrm{~T} 790 \mathrm{M}$ point mutation, exon 20 insertion, exon $21 \mathrm{~L} 858 \mathrm{R}$ point mutation, and exon $21 \mathrm{~L} 861 \mathrm{Q}$ point mutation). The result showed that all of above detections were negative.

\section{Discussion}

Surgical resection is the main treatment for lung cancer patients, especially for the patient in early stage, but patients with resected early stage had high recurrence rate and low long-term survival rate $^{[6]}$. Lymph node metastasis is a big threat for early stage patients ${ }^{[7]}$. The management of these patients is difficult due to that available therapeutic schedule is limited. So understanding the mechanism of disease occurrence and development is important for us.

In this case, the patient experienced lobectomy and adjuvant chemotherapy, but he had regional recurrence with multiple swollen lymph nodes in mediastinum. The outcome of patients with lung cancer metastasis remains dismal. Even if the patients received comprehensive treatment, their prognosis did not improve significantly ${ }^{[8]}$. So far, however, this patient has survivaled for nearly 4 years. The patient has been receiving chemotherapy without the indication for surgery.

Recently, the research reports focused on the mechanism and cases of histologic transformation of lung cancer have become 


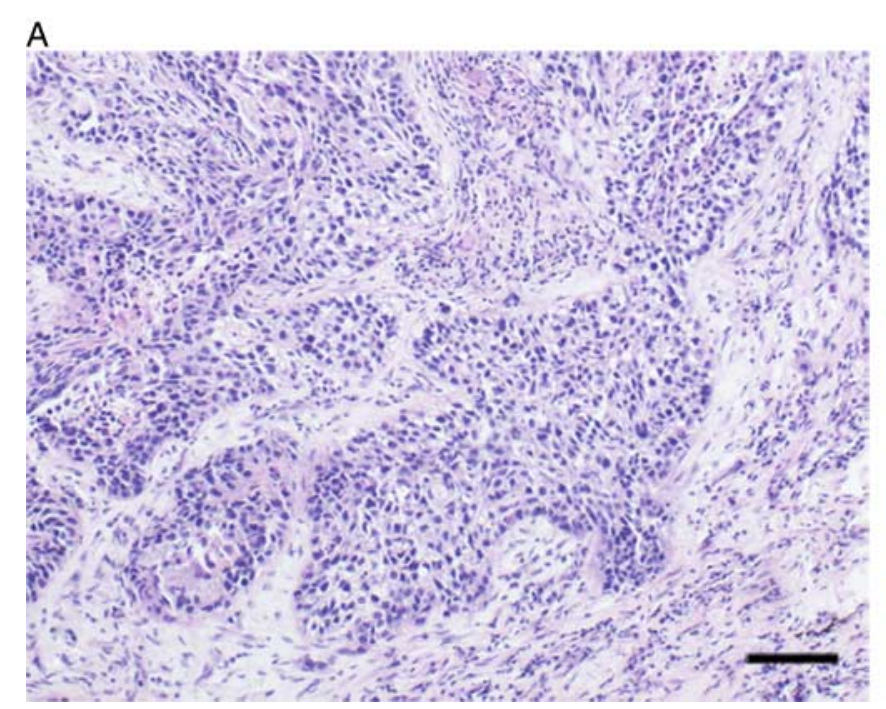

B

Figure 2. Histopathologic findings: squamous cell carcinoma (A, B). Bar $=400 \mu \mathrm{m}$.
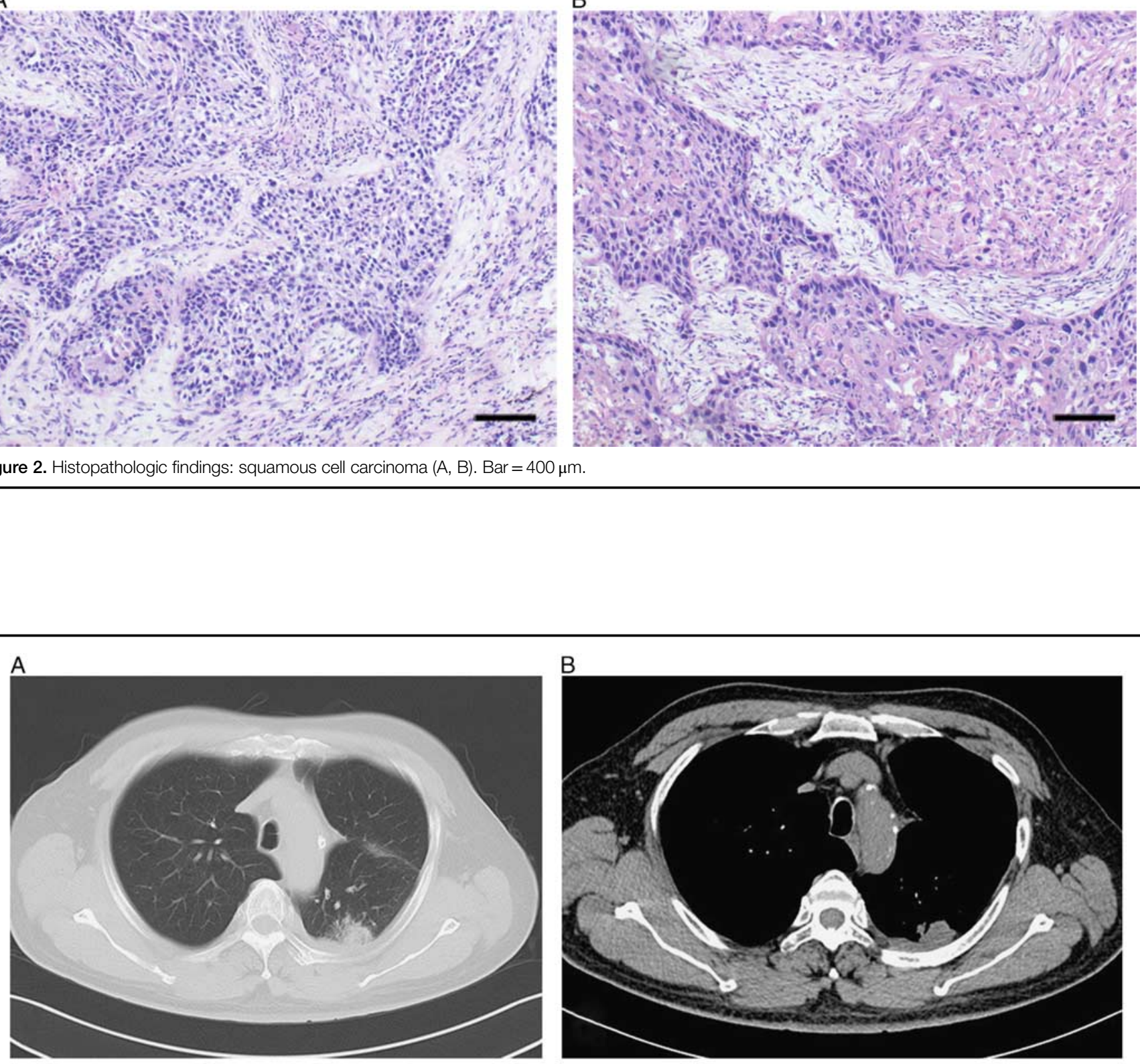

C

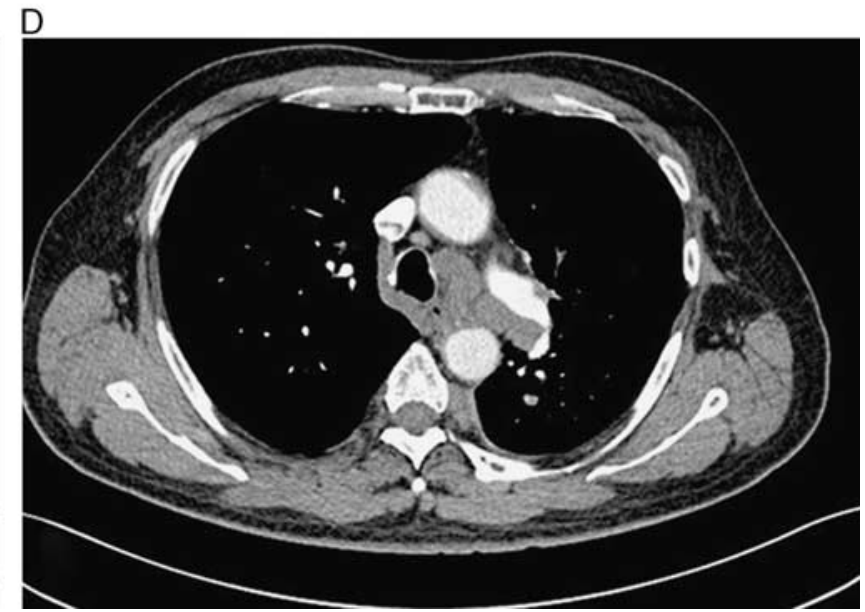

Figure 3. Computed tomography scan: (A, B) the irregular mass shadow in left lung; (C, D): the enlargement of mediastinal lymph nodes. 

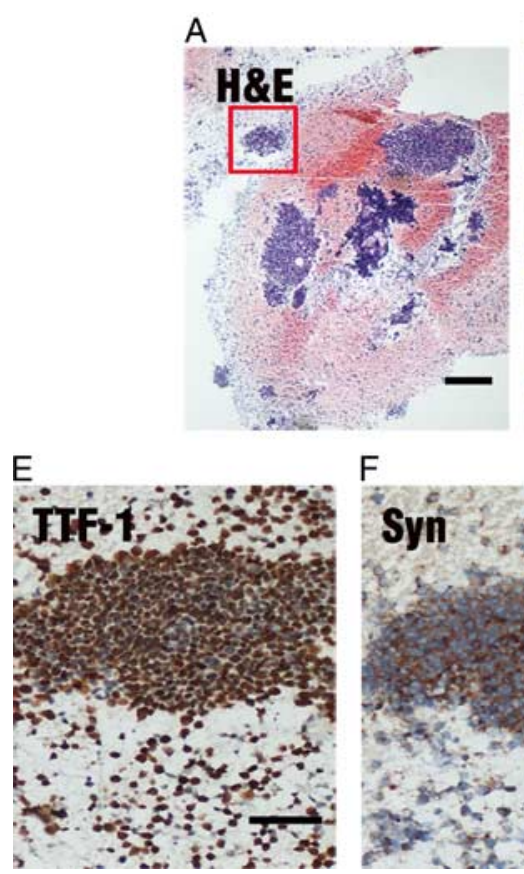

$\mathrm{F}$
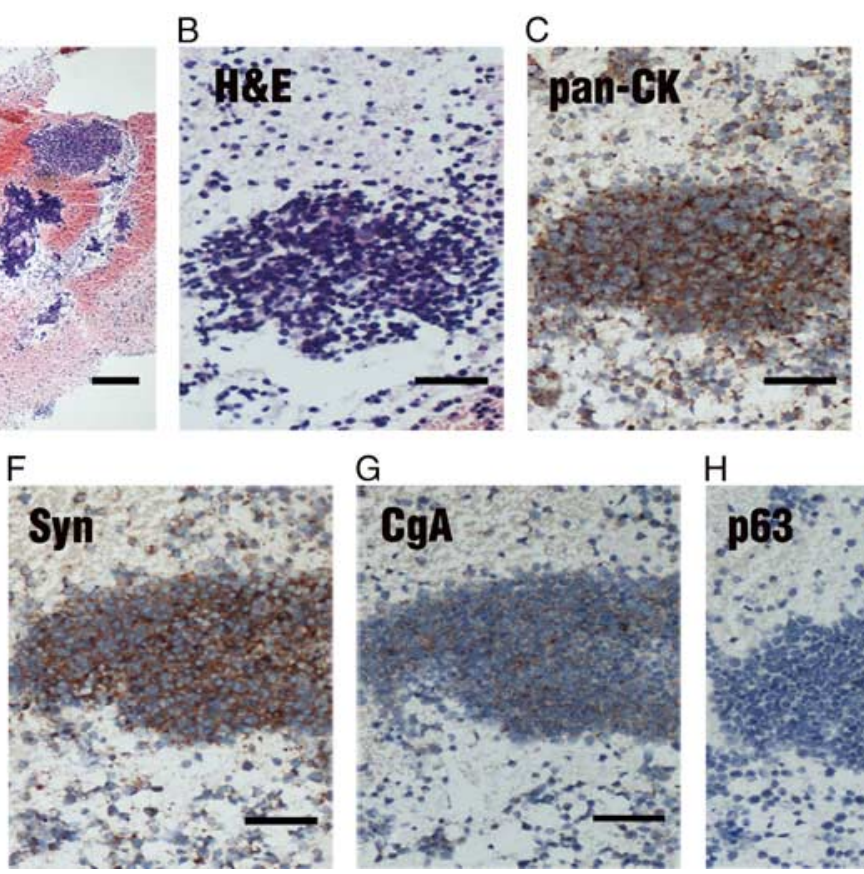

$\mathrm{H}$
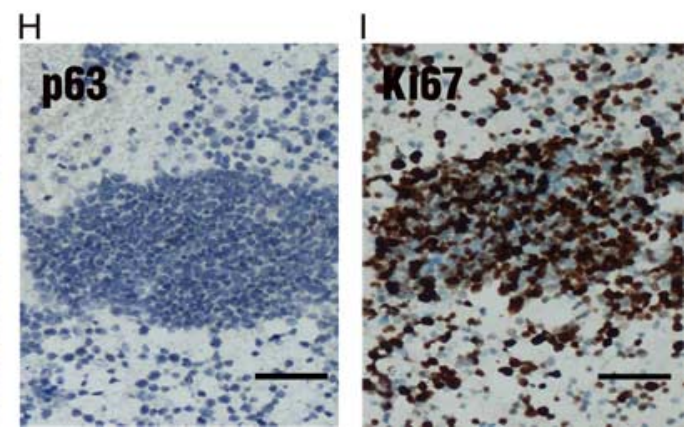

Figure 4. Histopathologic findings: small cell lung cancer (A, B, H\&E); immunohistochemistry examination of pan-CK (C), CD56 (D), TTF-1(E), Syn (F), CgA (G), p63 $(H)$, and Ki67 (I). The serial area in (B-I) is enlarged area in red box of $(A)$. Bar $=80 \mu \mathrm{m}(A)$. Bar $=400 \mu \mathrm{m}(B-I)$.

more and more. The histologic transformation of lung cancer was firstly reported by Sequist et al ${ }^{[9]}$. The most frequently histologic transformation occurred to the patient with drug-resistant lung cancer due to EGFR mutations. In some case reports, histologic transformation from lung adenocarcinoma to squamous cell carcinoma is reported to be associated with the resistance to the EGFR-TKIs ${ }^{[10,11]}$.

Intracellular signaling pathways are widely involved in development, inflammation and carcinogenesis. For example, Wnt pathway activation is important for malignant transformation of liver progenitor cell. Histologic transformation of lung cancer is also involved in a series of signaling pathways ${ }^{[12]}$. Lung adenocarcinoma to squamous cell carcinoma transformation is correlated to the PI3K/AKT/mTOR pathway, in addition to the initial EGFR mutation, genomic alterations in PTEN, LKB1, PIK3CA ${ }^{[13]}$. The SCLC component arising from NSCLC component is dependent on the activation of the ASCL1 and PI3K/ AKT1 signaling pathways ${ }^{[14]}$. However, there was no gene mutation (including EGFR, ALK, BRAF V600E, etc.) in our case with histologic transformation from squamous cell carcinoma in primary focus to SCLC in metastatic lymph node lesion. This is a very unusual report. The reason might be the heterogeneity of cancer cells or drug-induced selection mechanisms ${ }^{[15,16]}$.

In summary, the therapeutic strategy of regional recurrent and lymph node metastatic lung cancer is a challenging problem. Despite complete surgical resection and adjuvant treatment, tumor histology transformation with recurrence and lymph node metastasis is still occur. We described the clinical characteristics, radiologic features, gene detection, histologic features, and immunohistochemical testing of a lung cancer patient with histologic transformation from primary focus to lymphatic metastasis. It requires us to further understand the pathogenesis of metastatic progression that appear to potential therapeutic target for optimizing treatment measures to malignant tumors.

\section{Ethical approval}

This study is approved by the Institute Research Ethics Committee (No. 2016 [LS] 014, China Medical University).

\section{Sources of funding}

The author(s) disclosed receipt of the following financial support for the research, authorship, and/or publication of this article: This work was supported by the National Natural Science Foundation of China [No. 81602022]; and the National Training Program of Innovation and Entrepreneurship for Undergraduates of China Medical University in 2019 [No. 201910159201].

\section{Authors' contributions}

H.Z. and G.W.: study design. X.Z. and Y.Q.: data analysis. H.Z., X.Z., and Y.Q.: funding acquisition. D.Z., Y.H., and H.Z.: writing —original draft. H.Z. and G.W.: writing—review and editing.

\section{Conflict of interest disclosures}

The authors declare that they have no financial conflict of interest with regard to the content of this report.

\section{Research registration unique identifying number (UIN)}

None. 


\section{Guarantor}

None.

\section{References}

[1] Kiankhooy A, Taylor MD, LaPar DJ, et al. Predictors of early recurrence for node-negative $\mathrm{t} 1$ to $\mathrm{t} 2 \mathrm{~b}$ non-small cell lung cancer. Ann Thorac Surg 2014;98:1175-83.

[2] Lou F, Huang J, Sima CS, et al. Patterns of recurrence and second primary lung cancer in earlystage lung cancer survivors followed with routine computed tomography surveillance. J Thorac Cardiovasc Surg 2013;145:75-82.

[3] Wang X, Yan S, Lv C, et al. Impact of omission of intrapulmonary lymph node retrieval on outcome evaluation of lung cancer patients without lymph node metastasis: a propensity score matching analysis. Clin Lung Cancer 2017;18:e411-16.

[4] Lee JK, Lee J, Kim S, et al. Clonal history and genetic predictors of transformation into small-cell carcinomas from lung adenocarcinomas. J Clin Oncol 2017;35:3065-74.

[5] Agha RA, Borrelli MR, Farwana R, et al. The SCARE 2018 Statement: updating consensus surgical case report (SCARE) Guidelines. Int J Surg 2018;60:132-6.

[6] Belanger AR, Hollyfield J, Yacovone G, et al. Incidence and clinical relevance of non-small cell lung cancer lymph node micro-metastasis detected by staging endobronchial ultrasound-guided transbronchial needle aspiration. J Thorac Dis 2019;11:3650-8.

[7] Li N, Tan F, Li J, et al. Blind spot in lung cancer lymph node metastasis: cross-lobe peripheral lymph node metastasis in early stage patients. Thorac Cancer 2018;9:480-5.
[8] Lafitte C, Etienne-Mastroianni B, Fournel C, et al. Implementation of optimized supportive care and hospital needs along the management of patients with advanced lung cancer. Lung Cancer 2018;124:143-7.

[9] Sequist LV, Waltman BA, Dias-Santagata D, et al. Genotypic and histological evolution of lung cancers acquiring resistance to EGFR inhibitors. Sci Transl Med 2011;3:75ra26.

[10] Haratani K, Hayashi H, Watanabe S, et al. Two cases of EGFR mutationpositive lung adenocarcinoma that transformed into squamous cell carcinoma: successful treatment of one case with rociletinib. Ann Oncol 2016;27:200-2.

[11] Izumi H, Yamasaki A, Ueda Y, et al. Squamous cell carcinoma transformation from EGFR-mutated lung adenocarcinoma: a case report and literature review. Clin Lung Cancer 2018;19:e63-6.

[12] Mokkapati S, Niopek K, Huang L, et al. $\beta$-catenin activation in a novel liver progenitor cell type is sufficient to cause hepatocellular carcinoma and hepatoblastoma. Cancer Res 2014;74:4515-25.

[13] Park S, Shim JH, Lee B, et al. Paired genomic analysis of squamous cell carcinoma transformed from EGFR-mutated lung adenocarcinoma. Lung Cancer 2019;134:7-15.

[14] Lin MW, Su KY, Su TJ, et al. Clinicopathological and genomic comparisons between different histologic components in combined small cell lung cancer and non-small cell lung cancer. Lung Cancer 2018;125: 282-90.

[15] Morgillo F, Della Corte CM, Fasano M, et al. Mechanisms of resistance to EGFR-targeted drugs: lung cancer. ESMO Open 2016;1: e000060.

[16] Iijima Y, Hirotsu Y, Mochizuki H, et al. Dynamic changes and druginduced selection of resistant clones in a patient with EGFR-mutated adenocarcinoma that acquired T790M mutation and transformed to small-cell lung cancer. Clin Lung Cancer 2018;19:e843-7. 